\title{
Pregnancy Rate after Tubal Reanastomosis: A Case Presentation and Systematic Review
}

\author{
Muhammad A Zucha ${ }^{1}$, Muhammad Lutfi ${ }^{2}$, Supak Silawani ${ }^{3}$, Anastasia Feliciana ${ }^{4}$, Pritania Astari ${ }^{5}$
}

\begin{abstract}
Tubal reanastomosis is an option to recanalize fallopian tubes after previous sterilization. Several factors may be important in defining the success rate afterwards. This paper aimed to describe our experience performing tubal reanastomosis, and discuss the fertility outcome through a systematic literature review. We successfully performed tubal reanastomosis that resulted in pregnancy within 1 year after the procedure. Microsurgical tubal reanastomosis was performed with four-stitch technique. In addition, we conducted systematic searching to describe the efficacy of tubal reanastomosis in reversing the fertility status. MEDLINE database was searched with keywords: tubal reanastomosis, sterilization, and fertility. According to our criteria and search protocol, 16 studies were found, including 8,584 subjects. Data of pregnancy rate of those studies were collected to determine a cumulative pregnancy rate. According to our systematic review, we found tubal reanastomosis may result in successful conception of $70.27 \%$ of patients. Moreover, microsurgical tubal reanastomosis has comparable outcome in comparison to minimal invasive surgical approach. Therefore, tubal reanastomosis with microsurgery may be provided to reverse fertility status in low-resource settings. Our results mainly impact on patients who underwent tubal sterilization but need a fertility reversal. Our shared technique may be used as an important insight for the surgical approach. More importantly, pregnancy rates from the systematic review may be used as evidence-based prediction number of pregnancies.

Keywords: Female sterilization, Pregnancy rate, Tubal microsurgery, Tubal reanastomosis.

International Journal of Infertility and Fetal Medicine (2022): 10.5005/jp-journals-10016-1254
\end{abstract}

\section{BACKGROUND}

Sterilization is one of the preferred contraceptive techniques for couples who do not want more children. In addition, female sterilization is often indicated for women when pregnancy is contraindicated, for example, those who have Class Risk IV of a major cardiac event. Female sterilization is preferred since the fecundity loss is unrelated to hormonal change or sexual function compromise. ${ }^{1}$ Female sterilization represents around $19 \%$ of contraception use in developing countries. In Indonesia, with the increasing proportion of women aged 35-49 and an increasing number of contraceptive prevalence rates (CPR), the estimate of female sterilization needs is around 28 million women. ${ }^{2}$ However, in some patients who previously underwent female sterilization, due to several reasons they want to have children again. Accordingly, tubal reanastomosis is one possible way to conceive for women desiring fertility who previously underwent tubal sterilization. This method may be preferred especially in low-resource settings or in developing countries because of its cost-effectiveness and feasibility. ${ }^{2}$

Tubal reanastomosis is a microsurgical technique to recanalize fallopian tubes after previous sterilization. This procedure involves a loupe or microscope for magnifying the tubes during reanastomosis procedure. Minimally invasive laparoscopy or robotic-assisted surgery are other alternative ways available to perform tubal reanastomosis surgery. ${ }^{3}$ The outcome and success rate following tubal reanastomosis differ among patients, indicating several factors may affect the outcome. This study presents one report of a successful procedure of tubal reanastomosis. We also discuss the possible factors to improve the success rate of tubal reanastomosis. Systematic literature searching was done from the MEDLINE database.

Our case involves a patient who desired fertility even though she previously underwent tubal sterilization. We performed a

\footnotetext{
1,2Department of Obstetrics and Gynecology, Universitas Gadjah Mada, Sleman, Yogyakarta, Indonesia

${ }^{3}$ Department of Obstetrics and Gynecology, Universitas Palangkaraya, Palangkaraya, Central Kalimantan, Indonesia

${ }^{4,5}$ School of Medicine, Universitas Gadjah Mada, Sleman, Yogyakarta, Indonesia

Corresponding Author: Muhammad A Zucha, Department of Obstetrics and Gynecology, Universitas Gadjah Mada, Sleman, Yogyakarta, Indonesia, Phone: +62 81391664860, e-mail: ary_zucha@yahoo.co.id

How to cite this article: Zucha MA, Lutfi M, Silawani S, et al. Pregnancy Rate after Tubal Reanastomosis: A Case Presentation and Systematic Review. Int J Infertil Fetal Med 2022;13(1):28-33.

Source of support: Nil

Conflict of interest: None
}

laparotomy approach to identify the previously ligated fallopian tubes. Using a loupe, we performed resection on both proximal and distal ends of the dissected tubes. Interrupted sutures were done on the mesosalpinx, followed by four stitches on muscular layers at 6, 3, 9, and 12 o'clock positions. Tubal patency test was then performed to check the patency of both tubes. An irrigated methylene blue into both fimbriae tubae indicated the patency of both tubes. During the 12-month follow-up, our patient became pregnant and delivered spontaneously.

Tubal reanastomosis is an alternative to in vitro fertilization (IVF) for patients who desire fertility after tubal sterilization. Some reports have also mentioned the superiority of tubal reanastomosis, in terms of feasibility and cost-effectiveness. It is an important technique in a low-resource setting. However, this method is rarely performed and less taught in the fellowship training. Pregnancy rate and the possibility of ectopic pregnancy are the main concerns.

(c) The Author(s). 2022 Open Access This article is distributed under the terms of the Creative Commons Attribution 4.0 International License (https://creativecommons. org/licenses/by-nc/4.0/), which permits unrestricted use, distribution, and non-commercial reproduction in any medium, provided you give appropriate credit to the original author(s) and the source, provide a link to the Creative Commons license, and indicate if changes were made. The Creative Commons Public Domain Dedication waiver (http://creativecommons.org/publicdomain/zero/1.0/) applies to the data made available in this article, unless otherwise stated. 
Therefore, in this paper, we also performed a systematic review of studies conducted in the last 10 years that evaluated the fertility outcome of tubal reanastomosis.

\section{Case Description}

A 36-year-old woman came to consult concerning the possibility to get pregnant after undergoing tubal sterilization 5 years ago. She desired fertility after her remarriage. After being evaluated and the other causes of infertility were excluded, tubal reanastomosis was preferred. Informed consent was collected before performing the operation. Before surgery, we performed hysterosalpingography to identify the obstructed fallopian tubes.

We performed microsurgical tubal reanastomosis, utilizing fine sutures, and magnifying loupe. During the operation, identification of the ligated tubes was needed. We found both tubes were ligated at distal $1 / 3^{\text {rd }}$ of the tubes. Step-by-step procedures of surgery we conducted are mentioned below:

- Dissection toward both ends of the tubes was initiated before reanastomosis. Removal of the affected tissue was conducted carefully to conserve enough length of the tubes.

- A probe was gently inserted through distal end of the fimbriae, pulled through proximal segment of fallopian tube. We used a flexible stent from catheter No. 6.

- Suturing of the mesosalpinx was done initially before reanastomosis, utilizing 6-0 polypropylene sutures.

- Muscular layer of the fallopian tubes was sutured with a four-stitch technique (at 3, 6, 9, and 12 o'clock position) by interrupted suture with polypropylene suture no. 6-0. Sutures were conducted in the submucosal layer of fallopian tubes muscle, avoiding tubal mucosa when possible.

- Finally, serous layers of the tubes were also recovered with interrupted sutures.

- A tubal patency test was done by irrigating methylene blue dye, for which dye leakage through the abdominal ostium indicated patent tubes.

- After completion of the procedures, pelvis was irrigated with diluted Dexamethasone in normal saline (1:20) to prevent adhesion.

Illustrated figure of the operation is presented in Figure 1. After postoperative recovery, the patient was recommended to have contraception for 3 months. Within 12-month follow-up, the patient successfully became pregnant.

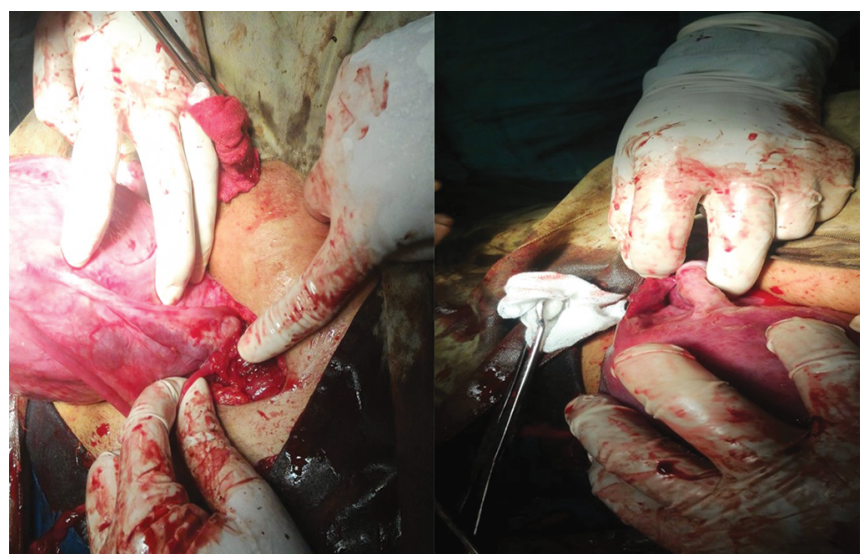

Fig. 1: Both tubes were obliterated at distal $1 / 3$ rd of the tubes (as pointed above). Both ovaries and fimbriae were normal

\section{Review Results}

Literature searching was performed using the PICO approach, which involved the clinical question: "How effective is tubal reanastomosis to recover fertility status of previously sterilized woman?" After developing this question, we managed to define the following:

P: tubal sterilized woman

I: tubal reanastomosis

C: without tubal reanastomosis

O: fertility rate (pregnancy rate and delivery rate)

We used PUBMED search engine to identify relevant literature on MEDLINE database. We used keywords: tubal reanastomosis, sterilization, and fertility. According to these keywords, the results were as follows:

From a total of 59 results from the database search, we selected studies based on the following eligibility criteria: the study must be conducted within the last 10 years, and studying the fertility outcome (pregnancy rate and/or delivery rate) of tubal reanastomosis after female sterilization. According to these criteria, a total of 16 studies were included in our review.

\section{Discussion}

Female tubal sterilization is a preferred contraceptive method in patients who do not intend to have children. It is the most common contraceptive used in women, and more than 180 million women have chosen this method. ${ }^{4}$ Tubal sterilization has a low failure rate ranging from $0.85-18.5 \%$ and a low overall complication rate. $^{5}$ It was first introduced through the laparotomy approach as the Pomeroy method to ligate and cut both fallopian tubes. Later, laparoscopic approach was developed to be less invasive via ligating devices, either electrocautery or application of fallopian rings or clips. More recently, hysteroscopic sterilization offers a lesser invasive approach. With the FDA approval to use nickel titanium (nitol) occlusion material in the tubal ostium in hysteroscopy, the application of hysteroscopic sterilization is becoming more popular. ${ }^{6}$

Globally, it is estimated that about $15 \%$ of women who had tubal sterilization regret their choice due to various reasons, for example, change of marital status, loss of the child, or change of attitude. $^{3}$ Accordingly, any possible way to restore the fertility status in females who underwent tubal sterilization is an important issue. ${ }^{7}$ Currently, the only options to reverse fertility status after tubal ligation are either IVF or tubal reanastomosis. Meanwhile, assisted reproductive technology through IVF is still a costly option. Across maternal age, tubal reanastomosis shows superior cost-effectiveness than IVF. Tubal reanastomosis is about 50\% more cost-effective than IVF. ${ }^{8}$ However, tubal reanastomosis is less frequently performed and is also less taught in fellowship training.

\section{Tubal Reanastomosis Surgical Technique}

Tubal surgery has evolved to be an effective technique in reproductive surgery. Successful tubal reanastomosis was initially performed in animal studies, prior to be conducted in patients by laparotomy. ${ }^{9}$ Classical technique of tubal reanastomosis occupies four muscular interrupted sutures and outer serosa layer sutures. Avoidance of suture on the mucosa layer is considered to improve pregnancy rate. ${ }^{10}$ Technique of tubal reapproximation is continuously improving. Technique utilizing fewer stitches was introduced, and even a single continuous suture also offered alternative option to the interrupted suture to reduce operation time without compromising tubal patency. ${ }^{11}$ Despite various 
techniques, the classical technique with four-stitch is still the most preferable technique. ${ }^{10}$

Laparoscopic approach facilitates image magnification that enables sutures in tubal reanastomosis, which is comparable to laparotomy with microscope. Various suture techniques in laparoscopic tubal reanastomosis have been described. The most common technique includes two sutures: at the mesosalpin $x$ and at the opposite that involves both muscular and the outer serosa layers. Dubuisson and Swolin introduced a single stitch technique in 1995 that only requires one suture to align two tubal segments. They performed suturing to the antimesial edge of the tube, connecting the layers of the serosa and musculature. This technique was designed to reduce the length of an operation procedure in laparoscopic tubal reanstomosis. ${ }^{12}$ Some authors also reported sutureless techniques. Automated clipping (autosuture) or tissue adhesive may be used in the laparoscopic sutureless approaches. Tulandi combined suturing technique to muscular layers with tissue adhesive to the serosa. The effectiveness was found similar to normal laparoscopic tubal reanastomosis using sutures to all layers. ${ }^{13}$ However, problems emerged in the application of tissue adhesive because some negative effects were reported in animal studies, such as dehiscence, fistulae, and intratubal adhesion. ${ }^{14}$

Robotic surgery is recently also an available approach in tubal reanastomosis. Two types of robots were applied in several case series: the da Vinci Surgical System and Zeus Robotic Surgical System with varying suture techniques and suture materials. Kavoussi reported advantages of robotic tubal reanastomosis, including shorter operative time, less technical difficulty due to enhanced ergonomics, and minimization of surgical fatigue. They performed a single-stitch technique, rather than a four-stitch technique that is more common. Dilute vasopressin was injected into mesosalpinx for hemostasis. After dissection of the occluded fallopian tubes, a double-needle 5-0 polyglactin suture was performed into the lumen at the 12-o'clock position from mucosa layer into muscularis and out through serosa layer. ${ }^{15}$

Reanastomosis requires a very thin suture material, and a loupe or microscopic surgical device is needed to assist suturing of the tubes. Microsurgical reanastomosis is also considered a good option, and even better than the laparoscopic approach. ${ }^{3}$ For several reasons, the laparoscopic approach is less applicable because it involves a steep learning curve. The other limitations of laparoscopic approach are tremor amplification and two-dimensional vision. This laparoscopic approach is also a very difficult task for reanastomosis due to a limited degree of freedom for dissection and suturing. ${ }^{16}$ Robot-assisted reanastomosis may reduce the tremor amplification and limited degree of freedom. ${ }^{17}$ With the precise assistance of the robotic device, tubal reanastomosis can also be performed with the single-stitch technique. ${ }^{15}$ However, there are cost concerns for robotic tubal reanastomosis. ${ }^{15,17}$

In our case, we successfully performed microsurgical laparotomy approach of tubal reanastomosis after identifying the

Table 1: Searching strategy and number of literatures found

\begin{tabular}{lc}
\hline \multicolumn{1}{c}{ Keywords } & $\begin{array}{c}\text { Number of } \\
\text { literature }\end{array}$ \\
\hline $\begin{array}{l}\text { (Tubal recanalization) OR reanastomosis } \\
\text { [(Tubal recanalization) OR reanastomosis] AND } \\
\text { sterilization }\end{array}$ & 442 \\
$\begin{array}{l}\text { [(Tubal recanalization) OR reanastomosis AND } \\
\text { sterilization] NOT vasectomy }\end{array}$ & 64 \\
\hline
\end{tabular}

(Table 1) ligated or dissected fallopian tubes. We used a stent from catheter No. 6 to facilitate tubal lumen patency while bringing the two tubal segments together. After removing the ligated tubes, we performed microsurgery for suturing. Mesosalpinx was sutured with polypropylene material no. 6.0. Further, muscular layers at the end of both tubes were identified and they were sutured systematically at $6,12,3$, and 9 o'clock with 6.0 polypropylene sutures. The serous layer was then sutured with nonlocking continuous sutures. Before we closed the abdomen, the tubal patency test by irrigating methylene blue dye was done to make sure that both tubes were patent and there was no leakage from the reanastomosis sutures. The illustrated figure of the technique is provided in Figure 2.

\section{Effectiveness of Tubal Reanastomosis}

Our study collected data within the last 10 years to provide evidence about the efficacy of tubal reanastomosis after tubal sterilization, in terms of pregnancy rate. Our results may be used to compare the efficacy of tubal reanastomosis, and provide evidence to convince clinicians to perform tubal reanastomosis.

We conducted a systematic literature search to find relevant studies in the last 10 years. We searched with the PUBMED search engine and identified 16 studies that met our eligibility criteria in MEDLINE. Among 8,584 subjects of the studies, we found that the pregnancy rate ranged between 44 and $85.1 \%$, disregarding the age of the patients. The mean of the pregnancy rate was $70.27 \%$. In addition, the mean delivery rate of the cumulated studies was $41.28 \%$. The largest study of laparoscopic tubal reanastomosis was performed by Feng et al. that had a pregnancy rate of $67.95 \%$ from 156 subjects. Unfortunately, there were no data provided for delivery rate and ectopic pregnancy rate. ${ }^{18}$ In the last 10 years, the pregnancy rate of laparoscopic tubal reanastomosis ranged between 52.11 and $75.3 \%{ }^{16,18-23}$ Cumulatively, laparoscopic tubal reanastomosis has a pregnancy rate of $67.68 \%$, delivery rate of $50.34 \%$, and ectopic pregnancy rate of $5.64 \%$ (Table 2 ).

Rates of pregnancy were very high in the microsurgical tubal reanastomosis studies conducted by Moon et al. and Berger et al. The Korean study by Moon et al. recruited a large number of subjects, with 961 women. However, that study did not provide the data of delivery rate. ${ }^{24}$ Meanwhile, Berger et al. recruited

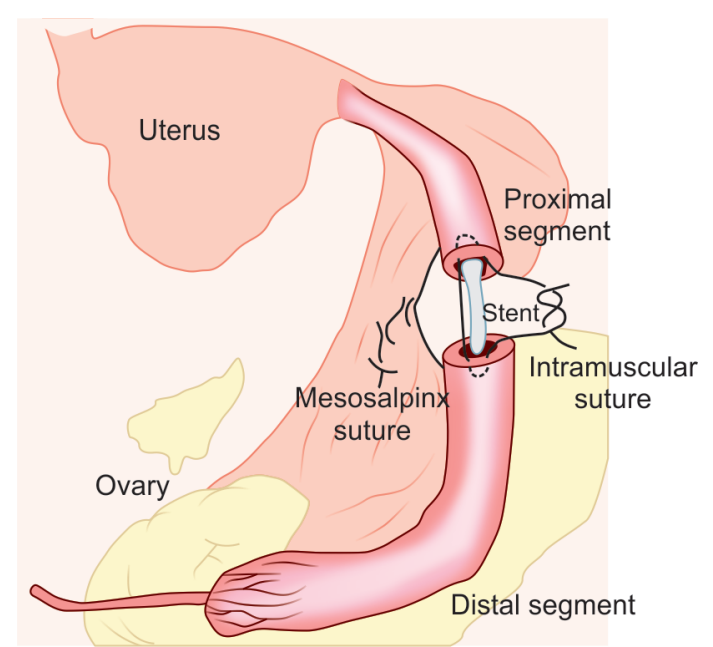

Fig. 2: Illustration figure of the microsurgery procedure. Interrupted muscular suture was performed after mesosalpinx suture and stent insertion 
Pregnancy Rate after Tubal Reanastomosis

Table 2: Cumulative pregnancy rate from 8,584 subjects who underwent tubal reanastomosis within the last 10 years

\begin{tabular}{|c|c|c|c|c|c|}
\hline Surgical approach & $\begin{array}{l}\text { Number of } \\
\text { cases }\end{array}$ & Pregnancy rate & Delivery rate & $\begin{array}{l}\text { Ectopic pregnancy } \\
\text { rate }\end{array}$ & Author, year \\
\hline Robotic surgery & 10 & $8(80 \%)$ & $7(70 \%)$ & $1(10 \%)$ & $\begin{array}{l}\text { (Kavoussi, Kavoussi and Lebovic, } \\
\text { 2014) }\end{array}$ \\
\hline \multirow[t]{2}{*}{ Robotic surgery } & 10 & $7(70 \%)$ & $5(50 \%)$ & $1(10 \%)$ & (Göçmen, Şanlikan and Uçar, 2013) \\
\hline & 20 & $15 / 20(75 \%)$ & $12 / 20(60 \%)$ & $2 / 20(20 \%)$ & \\
\hline Laparoscopy & 130 & $98(75.3 \%)$ & $68(52.7 \%)$ & $11(8.46 \%)$ & (Godin et al., 2019) \\
\hline Laparoscopy & 156 & $106(67.95 \%)$ & - & - & (Feng et al., 2019) \\
\hline Laparoscopy & 27 & $15(55.56 \%)$ & $14(51.85 \%)$ & $1(3.70 \%)$ & (Karayalcin et al., 2017) \\
\hline Laparoscopy & 71 & $37(52.11 \%)$ & $30(42.25 \%)$ & $2(2.82 \%)$ & (Paul, 2015) \\
\hline Laparoscopy & 88 & $58(65.91 \%)$ & $37(42.05 \%)$ & $6(6.82 \%)$ & (Van De Water et al., 2015) \\
\hline Laparoscopy & 29 & $17(58.8 \%)$ & - & - & (Jayakrishnan and Baheti, 2011) \\
\hline \multirow[t]{2}{*}{ Laparoscopy } & 127 & $94(74.01 \%)$ & $74(58.73 \%)$ & $5(3.93 \%)$ & (Schepens et al., 2011) \\
\hline & 628 & $425 / 628(67.68 \%)$ & $223 / 443(50.34 \%)$ & $25 / 443$ (5.64\%) & \\
\hline Microsurgery & 10 & $5(50 \%)$ & $5(50 \%)$ & $0(0 \%)$ & (Koteshwar and Siddesh, 2016) \\
\hline Microsurgery & 6,692 & $4,618(69 \%)$ & $2,314(34.58 \%)$ & $870(13 \%)$ & (Berger, Thorp and Weaver, 2016) \\
\hline Microsurgery & 961 & $817(85.1 \%)$ & $794(82.62 \%)$ & $24(2.5 \%)$ & (Moon et al., 2012) \\
\hline Microsurgery & 25 & $11(44 \%)$ & $10(40 \%)$ & $1(4 \%)$ & (Ramalingappa and Yashoda, 2012) \\
\hline Microsurgery & 140 & $62(44.29 \%)$ & $27(19.29 \%)$ & $14(10 \%)$ & (Hirth, 2010) \\
\hline Microsurgery & 19 & $14(73.68 \%)$ & $12(63.16 \%)$ & $4(21.05 \%)$ & (Tan and Loh, 2010) \\
\hline \multirow[t]{2}{*}{ Microsurgery } & 89 & $65(73.03 \%)$ & $45(50.56 \%)$ & $6(6.74 \%)$ & (Schippert et al., 2010) \\
\hline & 7,936 & $\begin{array}{c}5,592 / 7,936 \\
(70.46 \%)\end{array}$ & $\begin{array}{c}3,207 / 7,936 \\
(40.41 \%)\end{array}$ & $\begin{array}{c}919 / 7,936 \\
(11.58 \%) \\
\end{array}$ & \\
\hline Cumulative & 8,584 & $\begin{array}{c}6,032 / 8,584 \\
(70.27 \%)\end{array}$ & $\begin{array}{c}3,442 / 8,339 \\
(41.28 \%)\end{array}$ & $\begin{array}{c}946 / 8,399 \\
(11.34 \%)\end{array}$ & \\
\hline
\end{tabular}

an even larger number of subjects. They studied the fertility outcome of microsurgical tubal reanastomosis of 6,692 subjects and found a pregnancy rate of around $69 \%$. Moreover, they also mentioned a delivery rate of $34.58 \%$ and ectopic pregnancy rate of $13 \% .{ }^{25}$ Interestingly, our cumulative evidence did not show any difference of pregnancy rate $(p=0.36)$ and delivery rate $(p=0.74)$ among different surgical approach. Therefore, microsurgery has comparable effectiveness to minimal invasive surgery in reversing fertility status after tubal sterilization (Fig. 3).

In addition to the pregnancy and delivery rates, some studies also mentioned the possible complications, especially the incidence of ectopic pregnancy. ${ }^{26-28}$ It is understandable that reanastomosis may alter the passage of oocyte or the conception product into the uterine cavity. Among 8,339 subjects from 14 studies that evaluated the incidence of ectopic pregnancy, 946 cases were found. Thus, the ectopic pregnancy rate was $11.34 \%$. Ectopic pregnancy rate did not differ significantly among the different surgical approaches of tubal reanastomosis $(p=0.51)$. Location of anastomosis is associated with the incidence of ectopic pregnancy. When anastomosis is done at an interstitial-ampulla site, it may have higher probability for ectopic pregnancy rather than any other site of anastomosis. Incidence of ectopic pregnancy in interstitial-ampulla anastomosis was around $20.0 \%$, in comparison to $2.49 \%$ of overall ectopic pregnancy incidence. ${ }^{24}$

Some studies were conducted not only to evaluate the pregnancy outcome, but also to identify the possible factors that affect the pregnancy rate. One study including 71 subjects conducted by Paul et al. also evaluated the association with age, duration between sterilization-to-anastomosis, type of sterilization, and length of tube after reanastomosis. However, only length of remaining tube (more than $5 \mathrm{~cm}$ ) was significant as a predictor for pregnancy outcome $(p=0.0056){ }^{20}$ In contrast, the remaining factors were not associated with pregnancy rate. This finding was relevant with the results of the study conducted by Jayakrishnan et al. in 2011, that found none of women $(n=29)$ from the subjects who had remaining tubal length less than $5 \mathrm{~cm}$ could conceive. ${ }^{22}$ Therefore, tubal length after reanastomosis is an important parameter to restore fertility status.

Even though age was not a significant factor for pregnancy rate in the study conducted by Paul et al., several studies mentioned that age may be an important variable for pregnancy rate after tubal reanastomosis. The large study by Moon et al. that included 961 women found that the pregnancy rate was higher in the group with younger age. The pregnancy rate was $90.3 \%$ in women aged $<40$ years old, while pregnancy rate was lower in the older group. Pregnancy rate of the group aged $>40$ years old was only $53.9 \% .{ }^{24}$ This result indicates that patients in younger age may have better prognosis to reverse their fertility status after tubal reanastomosis.

Surgical technique is also a point of consideration. Reanastomosis procedure has evolved since first introduced as macrosurgery reanastomosis, and then involving loupe or microsurgical reanastomosis. Laparoscopic approach has also recently improved into robotic-assisted reanastomosis to improve accuracy of the operation. However, according to our pooled data (Table 2), the pregnancy rate did not show any marked difference among various operation approaches. The more important factor is the sterilization procedure before. Pregnancy rate in women who underwent sterilization with the Pomeroy method had lower 

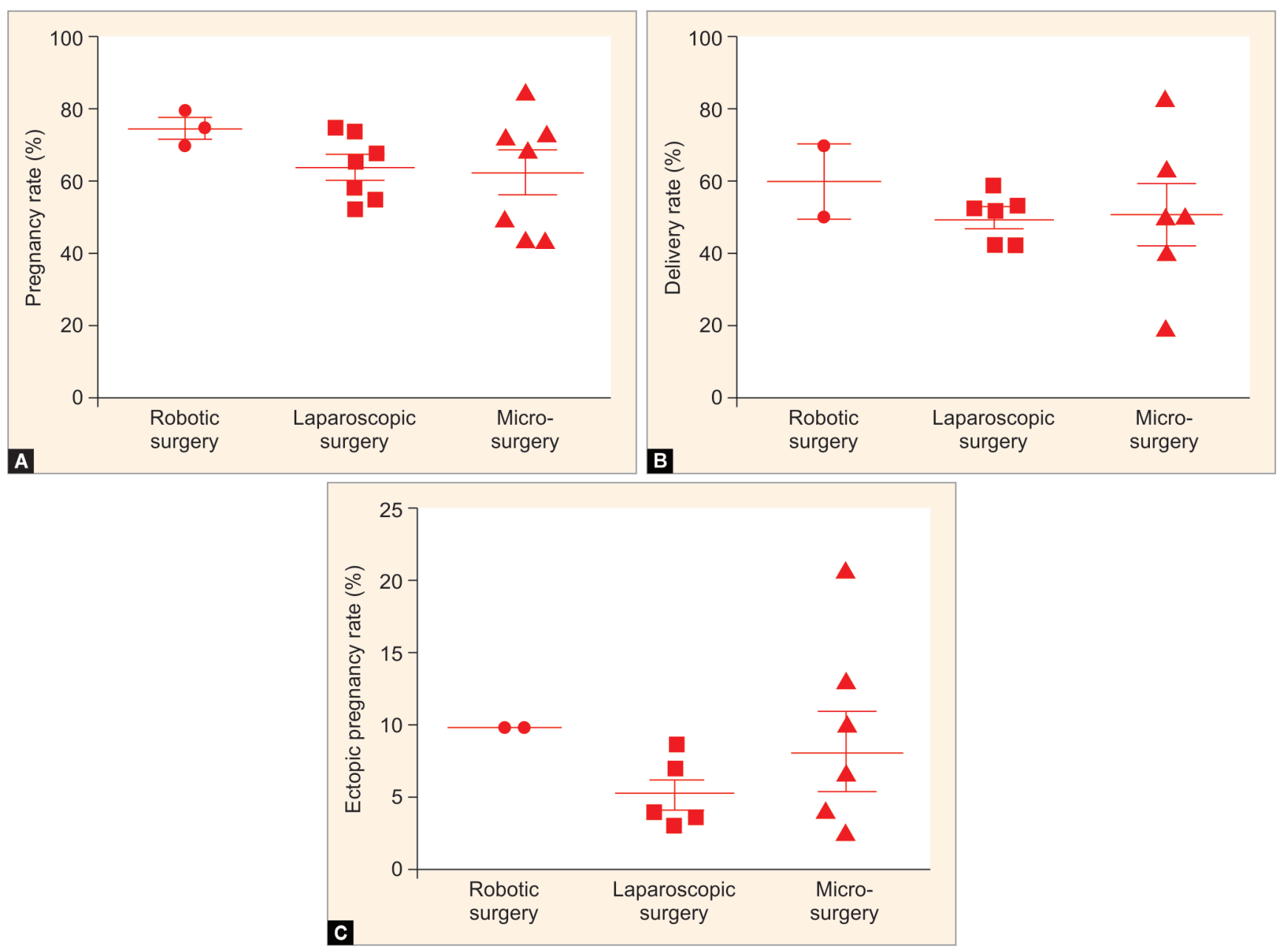

Figs 3 A to C: Pregnancy rate (A), derlivery rate (B) and ectopic pregnancy rate (C) among different surgical approach of tubal reanastomosis

pregnancy rate $(40 \%)$, in comparison to those who underwent laparoscopic tubal ligation (85.1\%). ${ }^{22}$

\section{Conclusion}

Microsurgical reanastomosis is a feasible and reliable method of tubal reanastomosis after female sterilization. Our presented case represents the applicability of the technique with four-stitch sutures. Microsurgical reanastomosis is not an inferior method compared to laparoscopic or robotic-assisted reanastomosis, and may be applied in lower-resource settings. Our systematic review mentioned that the pregnancy rate of tubal reanastomosis is as high as $70.27 \%$ with a delivery rate of $41.28 \%$. Several factors may improve the pregnancy rate after tubal reanastomosis. Younger age and tubal length (more than $5 \mathrm{~cm}$ ) might improve the pregnancy rate, so they should be considered in performing tubal reanastomosis.

\section{Clinical Significance}

Our results indicated that tubal reanastomosis is a feasible approach to restore the fertility status of the woman, as long as there are no other fertility problems. Our patient experienced a successful pregnancy within 1 year after four-stitch microsurgical tubal reanastomosis. From the systematic review conducted in this study, effectiveness of microsurgery is comparable to the more advanced approaches. Therefore, application of microsurgical tubal reanastomosis is not only feasible but also effective in reversing fertility status after tubal sterilization. Fertility restoration after female sterilization is not a myth, it is a hope.

\section{References}

1. Gizzo S, Bertocco A, Saccardi C, et al. Female sterilization: update on clinical efficacy, side effects and contraindications Minim Invasive Ther Allied Technol 2014;23(5):261-270. DOI: 10.3109/13645706.2014.901975

2. Hull TH, Mosley H. Revitalization of family planning in Indonesia. 2009;94.

3. RamalingappaA, Yashoda. A study on tubal recanalization. J Obstet Gynecol India 2012;62(2):179-183. DOI: 10.1007/s13224-012-0165-5

4. Beerthuizen R. State-of-the-art of non-hormonal methods of contraception: V. Female sterilisation. Eur J Contracept Reprod Heal Care 2010;15(2):124-135. DOI: 10.3109/13625181003597037

5. Varma R, GuptaJK. Failed sterilisation: evidence-based review and medico-legal ramifications. BJOG 2004;111(12):1322-1332. DOI: 10.1111/j.1471-0528.2004.00281.x

6. Murthy P, Edwards J, Pathak M. Update on hysteroscopic sterilisation. Obstet Gynaecol 2017;19(3):227-235. DOI: 10.1111/tog.12390

7. Koteshwar S, Siddesh A. A study of tubal recanalization in Era of ART (assisted reproduction technology). J Clin Diagnostic Res 2016;10(2):QC01-QC03. DOI: 10.7860/JCDR/2016/17376.7243

8. Messinger LB, Alford CE, Csokmay JM, et al. Cost and efficacy comparison of in vitro fertilization and tubal anastomosis for women after tubal ligation. Fertil Steril 2015;104(1):32-38.e4. DOI: 10.1016/j. fertnstert.2015.04.019 
9. Caballero-Gómez JM, Ortega-Moreno J. Study of two simplified microsurgical techniques for uterine horn anastomosis in rat. Arch Gynecol Obstet 1993;252(4):191-195. DOI: 10.1007/BF02426357

10. Estes SJ, Bhagavath B, Lindheim SR. Tubal anastomosis: once in a blue moon? Fertil Steril 2018;110(1)64-65. DOI: 10.1016/j. fertnstert.2018.03.024

11. Hurwitz A, Amir G, Yagel S, et al. A single continuous suture as a possible alternative to the interrupted suture for tubal anastomosis. Int J Infertil 1990;35(2):125-128.

12. Dubuisson JB, Swolin K. Surgery: brief communication: laparoscopic tubal anastomosis (the one stitch technique): preliminary results. Hum Reprod 1995;10(8):2044-2046. DOI: 10.1093/oxfordjournals. humrep.a136232

13. Tulandi T. Effects of fibrin sealant on tubal anastomosis and adhesion formation. Fertil Steril 1991;56(1):136-138. DOI: 10.1016/s00150282(16)54432-x

14. Gauwerky JFH, Klose RP, Forssmann WG. Surgery: fibrin glue for anastomosis of the fallopian tube-morphology. Hum Reprod 1993;8(12):2108-2114. DOI: 10.1093/oxfordjournals. humrep.a137991

15. Kavoussi SK, Kavoussi KM, Lebovic DI. Robotic-assisted tubal anastomosis with one-stitchtechnique.J RobotSurg 2014;8(2):133-136. DOI: 10.1007/s11701-013-0442-z

16. Godin PA, Syrios K, Rege G, et al. Laparoscopic reversal of tubal sterilization; a retrospective study over 135 cases. Front Surg 2018;5:79. DOI: 10.3389/fsurg.2018.00079

17. Göçmen A, Şanlikan F, Uçar MG. Robot-assisted tubal reanastomosis Initial experience in a single institution. Taiwan J Obstet Gynecol 2013;52(1)77-80. DOI: 10.1016/j.tjog.2012.01.036

18. Feng $\mathrm{Y}$, Zhao $\mathrm{H}, \mathrm{Xu} \mathrm{H}$, et al. Analysis of pregnancy outcome after anastomosis of oviduct and its influencing factors. BMC Pregnancy Childbirth 2019;19(1):1-5. DOI: 10.1186/s12884019-2469-2
19. Karayalcin R, Ozcan S, Tokmak A, et al. Pregnancy outcome of laparoscopic tubal reanastomosis: retrospective results from a single clinical centre. J Int Med Res 2017;45(3):1245-1252. DOI: 10.1177/0300060517709815

20. Paul P,Bhosale $S A$, Khan $S$, et al. Fertility outcome in laparoscopic single tube reanastomosis. J Reprod Med 2015;60(1-2):30-36.

21. Van De Water M, Bosteels J, De Sutter $P$, et al. Laparoscopic non-microsurgical tubal reanastomosis: a retrospective cohort study. Eur J Contracept Reprod Heal Care 2015;20(3):193-200. DOI: 10.3109/13625187.2014.990087

22. Jayakrishnan K, Baheti SN. Laparoscopic tubal sterilization reversal and fertility outcomes. J Hum Reprod Sci 2011;4(3):125-129. DOI: 10.4103/0974-1208.92286

23. Schepens JJ, Mol BW, Wiegerinck MA, et al. Pregnancy outcomes and prognostic factors from tubal sterilization reversal by sutureless laparoscopical re-anastomosis: a retrospective cohort study. Hum Reprod 2011;26(2):354-359. DOI: 10.1093/humrep/deq326

24. Moon HS, Joo BS, Park GS, et al. High pregnancy rate after microsurgical tubal reanastomosis by temporary loose parallel 4-quadrant sutures technique: a long long-term follow-up report on 961 cases. Hum Reprod 2012;27(6):1657-1662. DOI: 10.1093/humrep/des078

25. Berger GS, Thorp JM, Weaver MA. Effectiveness of bilateral tubotubal anastomosis in a large outpatient population. Hum Reprod 2016;31(5):1120-1125. DOI: 10.1093/humrep/dew038

26. Schippert C,Bassler C, Soergel P, et al. Reconstructive, organpreserving microsurgery in tubal infertility: still an alternative to in vitro fertilization. Fertil Steril 2010;93(4):1359-1361. DOI: 10.1016/j. fertnstert.2009.08.017

27. Hirth R, Zbella E, Sanchez M, et al. Microtubal reanastomosis: success rates as compared to in vitro fertilization. J Reprod Med 2010;55(3-4):161-165.

28. Tan HH, Loh SF. Microsurgical reversal of sterilisation - Is this still clinically relevant today? Ann Acad Med Singapore 2010;39(1): 22-26. 